Für die Grundversorger war und ist es wichtig, mehr als sechs Monate nach ihrer Kundgebung auf dem Bundesplatz langsam einige konkrete Ergebnisse ihrer Aktion zu sehen.

Der erste Ansatz einer Antwort auf diese ungeduldige Erwartung bestand in zwei Projekten der Schweizerischen Sanitätsdirektorenkonferenz. Diese sind Gegenstand des nachfolgenden Editorials und der vertieften Erläuterung auf Seite 1938 dieser Ausgabe der Schweizerischen Ärztezeitung.

Die Projekte wurden zwar vor dem 1. April ins Leben gerufen, aber die Stellung, die die Ärztinnen und Ärzte - vor allem die
Grundversorger - anschliessend bei ihrer Ausarbeitung einnehmen konnten, wurde ausserordentlich positiv, ja entscheidend beeinflusst und verstärkt.

Aus diesem Grund hat der Zentralvorstand der FMH mit grossem Vergnügen den Präsidenten des Kollegiums für Hausarztmedizin, Dr. med. Marc Müller, damit beauftragt, das Editorial dieser Ausgabe zu verfassen.

Dr. med. Jacques de Haller, Präsident der FMH

\title{
Stärkung der Grundversorgung
}

Vor fast fünfzig Jahren wurde ein Facharzttitel für Allgemeinmedizin geschaffen, mit dem Ziel, den Hausarzt den Spezialisten (damals «Spezialärzte» genannt) gleichzusetzen. Ziel war es, die medizinische Grundversorgung durch $60 \%$ Hausärzte sicherzustellen, die $40 \%$ Spezialisten sollten sich um die organspezifischen Details kümmern. Die Faszination der intellektuellen und technischen Spielereien, gepaart mit zunehmenden wirtschaftlichen Disparitäten, haben das geplante Gleichgewicht immer mehr zuungunsten der Grundversorger verschoben. Heute praktizieren nur noch knapp $40 \%$ Grundversorger - Tendenz sinkend - im Vergleich zu gut $60 \%$ Spezialisten.

Vor Jahren schon haben die Grundversorgergesellschaften (Allgemeinmediziner, Internisten und Kinderärzte) versucht, Gegensteuer zu geben - Stichworte «Tronc commun», GRAT-Verhandlungen -, aber leider vergeblich. Daraus folgte ein zunehmender Attraktivitätsverlust der Hausarztmedizin. Ausserdem verminderten ein Strukturwandel unserer Gesellschaft, lange Arbeitszeiten und Einzelkämpfertum in teils abgelegenen Randregionen den Stellenwert der Hausarztmedizin bei der Berufswahl unserer Nachfolgegeneration zunehmend. Dies alles liess uns schon vor fünf Jahren Artikel über einen bevorstehenden Hausärztemangel schreiben.

Mit einem Artikel in der «NZZ am Sonntag» im November 2004 begann dann auch die Öffentlichkeit, die Problematik bewusst wahrzunehmen. Während die eidgenössischen Politikerinnen und Politiker erst durch die Hausärztekundgebung vom 1 . April 2006 «geweckt» wurden, haben die kantonalen Gesundheitsdirektoren bereits im Herbst 2005 die Zeichen der Zeit erkannt, als erste Hausarztpraxen in ihren Kantonen nicht mehr besetzt werden konnten.

Plötzlich wurde die medizinische Grundversorgung zum Thema des «Dialoges der nationalen Gesundheitspolitik», der Plattform der Gesundheitsdirektorenkonferenz und der Eidgenossenschaft zur Steuerung unseres Gesundheitswesens. Die beiden Themen, die aufgrund ihrer Dringlichkeit als prioritär eingestuft wurden, entstammen unserem Forderungskatalog bei der Hausärztekundgebung: «Notfalldienst» und «Finanzierung der spezifischen Weiterbildung in den Hausarztpraxen» (Praxisassistenz).

In den Arbeitsgruppen, die sich während des Sommers mit diesen Themen beschäftigt haben, waren erstmals, so- weit ich mich erinnern kann, alle betroffenen Kreise vertreten: Repräsentanten des BAG, Vertreter der GDK und der kantonalen Gesundheitsbehörden, Delegierte der FMH, der kantonalen Ärztegesellschaften, Abgesandte des VSAO und von MWS (Medical Women Switzerland). Auch santésuisse sass am Tisch. Zum ersten Mal war es auch möglich, dass die Grundversorger als Direktbetroffene ihre Erfahrungen und Bedürfnisse direkt in die Diskussion einbringen konnten.

In einem bemerkenswert konstruktiven Klima des $\mathrm{Zu}$ hörens und Diskutierens entstanden die beiden Berichte, die in dieser Ausgabe der Schweizerischen Ärztezeitung abgedruckt sind.

Es ging dabei in erster Linie um eine Auslegeordnung des Status quo und der Problematik, aber in kurzer Zeit entstand bereits eine Vielzahl von Lösungsansätzen, die Ende Oktober auch vom obengenannten «Dialog Nationale Gesundheitspolitk» zur Kenntnis genommen wurden. Ein sehr gutes Resultat, auch wenn wir Grundversorger ungeduldig konkrete Massnahmen erwarten!

Der nächste Schritt folgt bei Erscheinen dieser Ausgabe: An der 8. Arbeitstagung «Nationale Gesundheitspolitik» werden 200 Expertinnen und Experten aus allen Sparten des Gesundheitswesens, Politikerinnen und Politiker, aber auch Ärztinnen und Ärzte - darunter wir, die Exponenten der Grundversorgergesellschaften - gemeinsam das weitere Vorgehen planen.

Mit Papieren löst man keine Probleme, auch mit interessanten Papieren nicht, gute Ideen schaffen keine neuen Hausärztinnen und Hausärzte. Taten sind gefragt.

Viele der nächsten Schritte können wir Ärztinnen und Ärzte nicht allein tun, wir sind auf Mithilfe angewiesen, auf Unterstützung von allen Akteuren im Gesundheitswesen, von Politikerinnen und Politikern, der ganzen Gesellschaft!

Denn: Medizinische Grundversorgung, Hausarztmedizin geht alle etwas an!

Unsere ärztliche Leistung wird immer mehr «gemessen». Wie misst man die Performance der Politiker/innen?

Dr. med. Marc Müller, Präsident des Kollegiums für Hausarztmedizin KHM 